\title{
Adverse event following immunization in a Nigerian tertiary health institution
}

\author{
${ }^{1} \mathrm{SA}$ Aderibigbe, ${ }^{1} \mathrm{GK}$ Osagbemi, ${ }^{2} \mathrm{OA}$ Bolarinwa \\ Dept of Epid \& Community Health, ${ }^{1}$ College of Health Sciences, University of Ilorin, Nigeria \\ ${ }^{2}$ University of Ilorin Teaching Hospital, llorin Nigeria \\ ABSTRACT
}

\begin{abstract}
Vaccine manufacturers develop products with the highest safety and effectiveness possible. But some very rare vaccine-related adverse events still occur. A descriptive retrospective study design was employed in this investigation. The investigation was limited to the four major health facilities in a metropolitan city that offer major immunization services to children. All cases of injection abscess or local reaction following vaccine administration from January 2004 to June 2006 were extracted from the records of each facility and included in the investigation. More than half of the cases reported were between $2-4$ months of age constituting $57.9 \%$ of all reported cases. The mean age of all reported cases was $4.368 \pm 2.794$ with a modal age of 2 months. Over half of all documented cases of adverse reactions following vaccine administration during the period under investigation were local swelling at site of injection $(50.9 \%)$. About half of all cases documented $(49.1 \%)$, were given DPT before development of adverse reactions. Almost all the cases of adverse reaction to vaccine administration (93\%) were seen at the tertiary health facility. Cold chain equipment should be supplied and maintained at utmost working conditions at all health facilities to guarantee potency of vaccines.
\end{abstract}

Keywords; Adverse Event Following Immunization, Epidemic, Investigation.

\section{INTRODUCTION}

One of the greatest achievements in public health has been infectious disease prevention through immunization. Very few interventions in the field of public health have been able to prevent as many deaths and diseases as vaccinations administered through organized immunization programs have done. Although the discovery and introduction of vaccines occurred at the end of the $18^{\text {th }}$ century, the surprising potential of vaccines was not truly recognized until 1977, when the eradication of smallpox was achieved (PAHO 2002).

Vaccines used in national immunization programmes are extremely safe and effective. However no biological or pharmaceutical product has yet been developed which is $100 \%$ safe and $100 \%$ effective (WHO, 2000; CDC, 1996). Vaccine manufacturers develop products with the highest safety and effectiveness possible, given current technology. But some very rare vaccine-related adverse events will always occur. Where surveillance is not adequate, these may not come to the notice of immunization staff or the public, but they nonetheless occur.

An adverse events following immunization (AEFI) is any adverse event that follows immunization that is believed to be caused by the immunization (WHO, 2002). Reported adverse events can either be true adverse events, i.e. really a result of the vaccine or immunization process, or coincidental events that are not due to the vaccine or immunization process but are temporally associated with immunization. Immunization can cause adverse events from the inherent properties of the vaccine (vaccine reaction), or some error in the immunization process (programme error). The event may occur after a vaccination has been given but is not caused by the vaccine or its administration (coincidental event). Anxiety-related reactions can arise from the fear or pain of the injection rather than the vaccine. In some cases the cause of the AEFI remains unknown (WHO, 2000; CDC, 1996; WHO, 2003).

Immunization safety; ensuring and monitoring the safety of all aspects of immunization, including vaccine quality, vaccine administration, and the disposal of used immunization injection equipment remains one of the challenges of national immunization programmes. Adverse events or rumours of adverse events following immunization are often poorly managed (WHO, 2003). If not rapidly and effectively dealt with, these events can undermine public confidence in immunization, and 
ultimately result in reduced immunization coverage and increased disease incidence (WHO, 2003).

This is a report of the investigation of an outbreak of adverse events following immunization at the Immunization clinic of a tertiary hospital located in the middle belt zone of Nigeria. The outbreak occurred between January and May 2006. Three other major health centres in the metropolis where immunization services were offered were also included in the investigation.

\section{MATERIALS AND METHODS}

A descriptive retrospective study design was employed in this investigation. The investigation was limited to the four major health facilities in the metropolis that offer immunization services to children.

All cases of injection abscess or local reaction following vaccine administration from January 2004 to June 2006 were extracted from the records of each facility and included in the investigation. Information obtained from each case identified included age, sex, vaccines taken, type of reaction documented, month and year the antigens was given. The batch numbers of all vaccines used in the facilities were also obtained from their records. The cold chain system and equipment at each health facility were also inspected. The technique of vaccine administration by the health workers was also observed.

The quantitative data obtained was fed into a computer and analysis was done with EPI- INFO version 6 software. The results were presented using frequency tables. Chi-square tests were done and Pvalue $<0.05$ was taken as statistically significant.

\section{RESULTS}

More than half of the cases reported were between 2 -4 months of age constituting $57.9 \%$ of all reported cases. This was followed by infants between $5-7$ months (19.3\%) and 8 - 10 months old infants $(17.6 \%)$. The mean age of all reported cases was $4.368 \pm 2.794$ with a modal age of 2 months (Table 1). A majority of cases were males accounting for $59.6 \%$ of all cases, while $40.4 \%$ were females. There was a statistically significant difference in the proportion of males affected compared to the females $(p<0.05)($ Table 1).

Over half of all documented cases of adverse reactions following vaccine administration during the period under investigation were local swelling at site of injection $(50.9 \%)$ followed by cellulites $(29.8 \%)$ and injection abscess (19.3\%) (Table 2). There was a statistically significant difference in the cases of local swelling recorded as at mid year 2006 as compared to all other adverse events $\mathrm{P}<0.05$ (Table 3 ).

Table 1: Demographic distribution of Patients

\begin{tabular}{|l|c|}
\hline Variables & Freq (\%) \\
\hline $\begin{array}{l}\text { Age (In Months) } \\
<2\end{array}$ & $3(5.3)$ \\
\hline $2-4$ & $33(57.9)$ \\
\hline $5-7$ & $11(19.3)$ \\
\hline $9-10$ & $10(17.6)$ \\
\hline $\begin{array}{l}\text { Sex } \\
\text { Male }\end{array}$ & $34(59.6)$ \\
\hline Female & $23(40.4)$ \\
\hline$X^{2}=4.25$ df $=1 \quad p=0.0393$ &
\end{tabular}

Table 2: Distribution of Patients by Diagnosis of AEFI and Vaccine Administered

\begin{tabular}{|l|r|}
\hline Variables & Freq (\%) \\
\hline Diagnosis & $17(29.8)$ \\
\hline Cellulitis & $11(19.3)$ \\
\hline Injection Abscess & $29(50.9)$ \\
\hline $\begin{array}{l}\text { Local swelling } \\
\text { Vaccine Administered }\end{array}$ \\
\hline BCG & $1(1.8)$ \\
\hline DPT & $28(49.1)$ \\
\hline HBV & $2(3.5)$ \\
\hline Measles & $4(7.0)$ \\
\hline Not Specified & $22(38.6)$ \\
\hline
\end{tabular}

Table 3: Number Of Cases Of Each Condition Seen Yearly

\begin{tabular}{|l|c|c|l|c|}
\hline Diagnosis & $\mathbf{2 0 0 4}$ & $\mathbf{2 0 0 5}$ & $\mathbf{2 0 0 6}$ & Total \\
\hline Cellulitis & 11 & 3 & 3 & 17 \\
\hline $\begin{array}{l}\text { Injection } \\
\text { Abscess }\end{array}$ & 4 & 3 & 4 & 11 \\
\hline Local swelling & 2 & 5 & 22 & 29 \\
\hline
\end{tabular}

About half of all cases documented (49.1\%), were given DPT before development of adverse reactions. 
Seven percent had been given measles vaccine. However $38.6 \%$ of them did not have records of which antigen was given (Table 2).

Half of all reported cases of adverse reactions to vaccine administration, 29 (50.9\%) where seen between January and May 2006 ; a period of 5 months as compared to 2004 when all the cases seen through out the year was $17(29.8 \%)$. In 2005, only 11 cases of AEFI were reported through out the year accounting for $19.3 \%$ of all cases been investigated (Table 3 ). On the distribution of cases recorded monthly, there is a marked increase in the reported cases of adverse reaction to vaccine administration in the months of February, March and May 2006 as compared to the same months of the preceding years. (Table 4)

Table 4: Number of Cases Seen Monthly

\begin{tabular}{|l|c|c|c|c|}
\hline & 2004 & 2005 & 2006 & Total \\
\hline January & 2 & 0 & 3 & 5 \\
February & 0 & 0 & 7 & 7 \\
March & 1 & 0 & 8 & 9 \\
April & 4 & 0 & 1 & 5 \\
May & 2 & 2 & 10 & 14 \\
June & 2 & 0 & 0 & 2 \\
July & 2 & 2 & 0 & 4 \\
August & 2 & 1 & 0 & 3 \\
September & 0 & 2 & 0 & 2 \\
October & 0 & 1 & 0 & 1 \\
November & 2 & 2 & 0 & 4 \\
December & 0 & 1 & 0 & 1 \\
\hline
\end{tabular}

Table 5: Cases Seen By Facility

\begin{tabular}{|l|l|}
\hline Facility & Frequency (\%) \\
\hline Facility A & $3(5.3)$ \\
Facility B & $1(1.8)$ \\
Facility C & $0(0)$ \\
Tertiary Facility & $53(93.0)$ \\
\hline
\end{tabular}

Chi square $=137.05 \mathrm{df}=3 \quad \mathrm{p}$ value $=0.0000$

Almost all the cases of adverse reactions to vaccine administration (93\%) were seen at the tertiary health facility. One other health facility, a Basic Health Centre accounted for $5.3 \%$ of all cases seen while
$1.8 \%$ of all cases where seen at the third facility; also a Basic health centre. It is noteworthy that no case was recorded at the fourth facility. There was a statistically significant difference in the number of cases seen at the tertiary centre when compared to the other immunization clinics $\mathrm{P}<0.05$ (Table 5).

In addition to the quantitative data obtained, it was discovered that all the four health facilities obtained their vaccine supply from the same source. Also the batch number of vaccines from all the facilities was the same for all antigens. However, at the immunization clinic of the tertiary centre, some vaccines did not tally with the others from the three other centres. Further questioning revealed that this particular batch of vaccines was sourced from centres outside the state.

The cold chain system and equipment were functioning at all the basic health facilities except the tertiary facility where their refrigerator was said to have broken down. Also, there were no functioning deep freezers at the immunization clinic of the tertiary health facility during the reported outbreak.

Another major finding was the fact that only the tertiary centre used the autodestruct syringe to administer the vaccines to the children. At all the centres, the health workers involved in administration claimed that the DPT, HBV and Yellow fever vaccines were given intramuscularly on the lateral aspect of the thigh, the BCG vaccine was administered intra-dermally at the left upper arm, while the Measles vaccine was given subcutaneously at the right upper arm. This fact was corroborated during observation of the health workers while giving the vaccines to the clients.

\section{DISCUSSION}

Adverse events following immunization (AEFI) are usually events that could be discomforting to children and could also undermine the success of the immunization programme due to its potential to encourage the spread of rumours and misconceptions among mothers. (Loevinsohn et al, 1990).

The first years of a child's life constitute the period of greatest vulnerability to diseases and of the first manifestations of other problems (developmental disorders, hearing impairments, and others). It is precisely during that period that vaccines are administered (PAHO, 2002). More than half $(57.9 \%)$ of the cases from this investigation were infants 
between $2-4$ months of age corroborating the vulnerability to disease theory from other authors.

The commonest AEFI discovered from this investigation was found to be local swelling at the site of injection accounting for about half $(50.9 \%)$ of all AEFI cases reported. This finding is in line with other studies which show the rate of local swelling following immunization to be between $40-80 \%$ (WHO, 2000; Scheifele et al 1994; Cherry, 1996). However, the fact that this finding was found to be statistically significant when compared to other AEFIs recorded in the same period and also when compared with same event recorded in previous years gives a cause for concern. In the same vein, the rate of occurrence of cellulites (29.8\%) and injection abscess (19.3\%) though within the limits reported by other authors in various studies (Cherry, 1996) were significantly higher than the occurrences reported within the same period in previous years (Tables 3,4 ). It may then be justifiable to say that an epidemic of AEFI had occurred in the reporting year (2006).

It is noteworthy that about half $(49.1 \%)$ of the cases of $\mathrm{AEFI}$ were recorded following immunization with DPT vaccine with a majority (93\%) of them significantly coming from the tertiary health facility (Table 5). This finding when evaluated against the backdrop that the tertiary hospital is expected to be well equipped in terms of personnel and logistics to deliver safe immunization services to the populace, makes it an issue for concern.

All the variables that could be postulated to have caused the outbreak vis-a-viz source of vaccines, batch number of vaccines used, cold chain equipment handling and maintenance as well as technique of administration of the antigens were found to be basically the same for all the facilities except the tertiary centre where it was discovered that vaccines were sometimes sourced from different sites outside the state when there were stock outs and that the cold chain equipment were not adequate. These factors may therefore be said to have been responsible for the outbreak of $A E F I$ in the health facility.
Health authorities are encouraged to ensure adequate supply of safe vaccines to all service delivery points to prevent stock outs of these vaccines which may then necessitate service providers to source for the vaccines from sources that can not be trusted. Also cold chain equipment should be supplied and maintained at utmost working conditions at all health facilities to guarantee the continuous potency of the vaccines.

\section{REFERENCES}

CDC (1996). Centre for Disease Control and Prevention Update: vaccine side effects, adverse reactions, contraindications, and precautions - recommendations of the Advisory Committee on Immunization Practices (ACIP). MMWR 1996; 45(No.RR-12): 1-35.

Cherry JD (1996). Historical review of pertussis and the classical vaccine. Journal of Infectious Diseases, 174, supt 3:S259-S263.

D W Scheifele DW, Bjornson G, Halperin SH, Mitchell L, and Boraston S (1994). Role of whole-cell pertussis vaccine in severe local reactions to the preschool (fifth) dose of diphtheria-pertussis-tetanus vaccine. CMAJ. 150(1): 29-35 PMCID: PMC1485779

Loevinsohn B, Garealla ET (1990). BCG ulcers and their effect on mother's willingness to allow their children receive other antigens. Trans $\mathrm{R}$ Soc Trop Med Hyg. 84(3):430. PMID: 2260181

Pan American Health Organization (2002). IMMUNIZATION SAFETY: How to address events allegedly attributable to vaccination or immunization? Washington, DC, United States.

WHO (2000). Expanded Program Immunization. Supplementary Information on vaccine safety, Part 2: "Background rates of adverse events Following Immunization", World Health Organization, WHO/V\&B/00.36, Geneva.

WHO (2002). Mass Measles Immunization Campaigns: Reporting and Investigating Adverse Events following Immunization.

WHO (2003). Weekly Epidemiological Record. 78, 41 - 48 